\title{
Comparison of grain to grain orientation and stiffness mapping by spatially resolved acoustic spectroscopy and EBSD
}

\author{
A.F. Mark ${ }^{1 *}$, W. Li $i^{2}$, S. Sharples ${ }^{2}$, P.J. Withers ${ }^{1}$
}

${ }^{1}$ School of Materials, University of Manchester, Grosvenor Street, Manchester M13 9PL, UK

${ }^{2}$ Applied Optics Group, University of Nottingham, University Park, Nottingham NG7 2RD, UK

*Corresponding author: a.mark@fkf.mpg.de

\begin{abstract}
Our aim was to establish the capability of spatially resolved acoustic spectroscopy (SRAS) to map grain orientations and the anisotropy in stiffness at the sub-mm to micron scale by comparing the method with electron backscatter diffraction (EBSD) undertaken within a scanning electron microscope. In the former the grain orientations are deduced by measuring the spatial variation in elastic modulus; conversely, in EBSD the elastic anisotropy is deduced from direct measurements of the crystal orientations. The two test-cases comprise mapping the fusion zones for large TIG and MMA welds in thick power plant austenitic and ferritic steels respectively; these are technologically important because, among other things, elastic anisotropy can cause ultrasonic weld inspection methods to become inaccurate because it causes bending in the paths of sound waves. The spatial resolution of SRAS is not as good as that for EBSD $(\sim 100 \mu \mathrm{m}$ versus $\sim$ a few $\mathrm{nm}$ ), nor is the angular resolution ( 1.5 degrees versus $\sim 0.5$ degrees). However the method can be applied to much larger areas (currently on the order of 300 $\mathrm{mm}$ square), is much faster ( $\sim$ times), is cheaper and easier to perform, and it could be undertaken on the manufacturing floor. Given these advantages, particularly to industrial users, and the on-going improvements to the method, SRAS has the potential to become a standard method for orientation mapping, particularly in cases where the elastic anisotropy is important over macroscopic/component length-scales.
\end{abstract}

key words: crystalline texture, weld microstructure, anisotropy, ultrasonic inspection, P91 ferritic steel, Inconel, SS 308, austenitic stainless steel 


\section{Introduction}

While for many engineering applications it is acceptable to assume that materials are homogeneous at the macroscale, in reality the individual polycrystal grains are highly anisotropic. This means that if there is significant preferred orientation (texture) then the bulk properties can be significantly anisotropic, both elastically and in terms of plastic behaviour. The inherent anisotropy of the individual grains also means that the direction-specific elastic modulus can be used to deduce the orientation of each grain if it can be sampled at a sufficiently local scale.

In this paper the capability of spatially resolved acoustic spectroscopy (SRAS) to map grain orientations at the micrometre scale is assessed by comparing the method with the now well established electron backscatter diffraction (EBSD) technique (Dingley, 1992; Schwarzer, 2009) undertaken within a scanning electron microscope. This comparison is carried out by examining the spatial variation in elastic modulus within the fusion zones of a tungsten inert gas (TIG) weld and a manual metal arc (MMA) weld in thick power plant steels. The elastic variation in the fusion zones of welds is technologically important because this anisotropy can cause ultrasonic weld inspection methods to become inaccurate because it causes bending in the paths of sound waves.

SRAS is a technique for mapping the surface acoustic wave velocity of a material. The spatially resolved acoustic spectroscopy technique has its roots in the optical spectroscopy technique, where by analysing a spectrum (of light) we can discover some of its properties (dominant wavelengths). In the acoustic sense we are analysing the spectrum of ultrasound emitted from a grating. Either (1) the frequency is fixed and the grating period is swept, or (2) the grating period is fixed and the frequency is swept. In this study the second of these two methods was used.

To acquire the acoustic spectrum for one 'point' on the sample, surface acoustic waves (SAWs) are excited by a broadband frequency source - in our case a pulsed laser using a grating of (fixed) regularly spaced lines. The SAWs are detected close to the grating by another laser a short distance away. The efficiency with which the SAWs are excited depends on how well the grating period matches the wavelength $(\lambda)$ of the SAWs at that frequency $(f)$. In this case, a frequency spectrum is generated and measured and the peak frequency is determined by Gaussian fitting. Once the peak frequency is known, then the SAW velocity can be calculated $(v=f \lambda)$, as the wavelength is determined by the fixed grating (Sharples, 2012; Li, 2012).

This is how we calculate the velocity in one direction for one 'point', where a point corresponds to the area under the grating. By scanning the excitation point across the sample, either by moving the laser system or by moving the sample, a map of location specific velocities is built up. To use SAW velocities in determining grain orientation they must be measured in multiple directions at each point. This is done by rotating either the measurement system or the material; in our case the measurement system was rotated. 
Previous work by Li et al. (Li, 2016) has demonstrated for a coarse-grained hexagonal polycrystal that velocity maps collected by SRAS correlate well with the c-axis grain orientation maps measured by EBSD. In this paper the velocity tensor is used to develop a stiffness map for direct comparison with the elastic anisotropy inferred from the crystal orientations measured by EBSD. Further the velocity map is used to infer the grain orientations for direct comparison with those measured by EBSD.

Electron backscattered diffraction can be considered as a benchmark for mapping grain orientations across an area. An electron beam within a scanning electron microscope impinges on a sample inclined at $70^{\circ}$. The diffracted beam is collected on a screen and shows Kikuchi bands that are characteristic of the angle of the illuminated grain to the sample surface. Software indexes the Kikuchi bands, identifying the relevant lattice planes, from which the orientation of the grain can be calculated. By scanning the beam over the sample a map of the grain orientations is built up revealing the grain structure with high spatial (a few $\mathrm{nm}$ at best) and high angular $\left(\sim 0.5^{\circ}\right)$ resolution.

In this study the capability of the SRAS method is validated by comparison to EBSD for mapping the stiffness in two large nuclear pressure vessel welds. One is a SS 308 weld joining two austenitic stainless steel plates by manual tungsten inert gas (TIG) welding. The second is a manual metal arc (MMA) weld joining P91 ferritic steel plates with a high nickel content weld filler (Inconel 600). Elastic anisotropy in such welds is an important consideration for the correction of ultrasound inspection data (Fan, 2013).

Austenitic stainless steel and Inconel 600 both have the FCC crystal structure, which is elastically anisotropic. The stiffest direction is along the $<111>$ direction and the softest direction is along the $<100>$ direction. In the solidification of these types of weld metals, grains nucleate at the boundary with the solid material and grow in a direction parallel to the thermal gradient. During weld solidification the crystals grow preferentially with $<100>$ directions parallel to the growth direction. When the parent metal also has the FCC structure growth is epitaxial, meaning the orientations of the growing crystals are influenced by the orientations of the grains on which they form. If there is no strong texture in the parent material, or if the parent material has a different structure (e.g. BCC steel such as P91), this growth behaviour would be expected to result in welds with essentially $<100>$ fibre textures locally oriented to match the thermal gradients imposed by the weld passes (Mark, 2014).

The anisotropy in the stiffness at the single crystal scale need not necessarily lead to anisotropy at the polycrystal (bulk) material scales. A polycrystal of FCC metal consisting of a sufficiently large number of grains of random texture would have essentially isotropic stiffness, whereas a fibre texture would confer transversely isotropic stiffness characteristics. In the case of FCC metals with $<100>$ fibre texture the softest direction is consistently parallel to the 'fibre' and the stiffnesses in the off-axis directions are averaged. 
Given that SRAS can map relatively large areas fairly quickly it could prove to be a useful tool in cases where the near surface or cross-sectional crystalline texture is of interest. Furthermore, given that it measures the crystalline anisotropy directly rather than inferring it from the grain orientations, SRAS may be especially well suited to problems where the elastic anisotropy is the critical parameter of interest, such as when undertaking ultrasound inspections of welds or considering the behaviour of aeroengine fan blades. Consequently the aim of this study is to compare SRAS with EBSD as a means of mapping elastic anisotropy and thereby grain orientations for relatively large samples of practical engineering interest.

\section{Experimental Method}

\subsection{Materials}

Two weld specimens were studied in this work. The first (denoted RR) was prepared and provided by Rolls-Royce Plc. This was a cross-section cut from a multi-pass weld using SS308 filler joining two $58 \mathrm{~mm}$ thick plates of austenitic stainless steel. The weld was laid down in three stages; the root passes (7 passes) were done using manual tungsten inert gas (TIG) welding. The bottom half of the main weld was done by automated orbital TIG welding; at the mid-point a flaw was introduced into the weld. Subsequently, the rest of the gap was filled, also with automated orbital TIG welding. The specimen was provided as a block approximately $60 \times 110 \times 50 \mathrm{~mm}^{3}$. In order to fit the sections into the SEM for EBSD, RR was first cut down to a slice $5 \mathrm{~mm}$ thick and then it was sectioned into 7 pieces each measuring approximately $15 \times 20 \mathrm{~mm}^{2}$. Each piece was ground and polished, finishing with vibration polishing with $0.06 \mu \mathrm{m}$ colloidal silica. Figure 1a shows a macrograph of a second slice of the same RR weld, sectioned only into two pieces.

The second weld specimen was provided by E.ON (denoted EON). This was a section cut from a circumferential, multi-pass, manual metal arc (MMA) weld joining two sections of P91 pipe. The pipes were $285 \mathrm{~mm}$ in diameter (OD) and $35 \mathrm{~mm}$ thick. This weld also had an initial root weld, done using TIG welding. High nickel content weld filler wires (TIG) and electrodes (MMA) were used. The EON specimen was provided as a slice $5 \mathrm{~mm}$ thick; it was cut in half, see Figure 1b. Each half was ground and polished in the same manner as the RR specimen. 

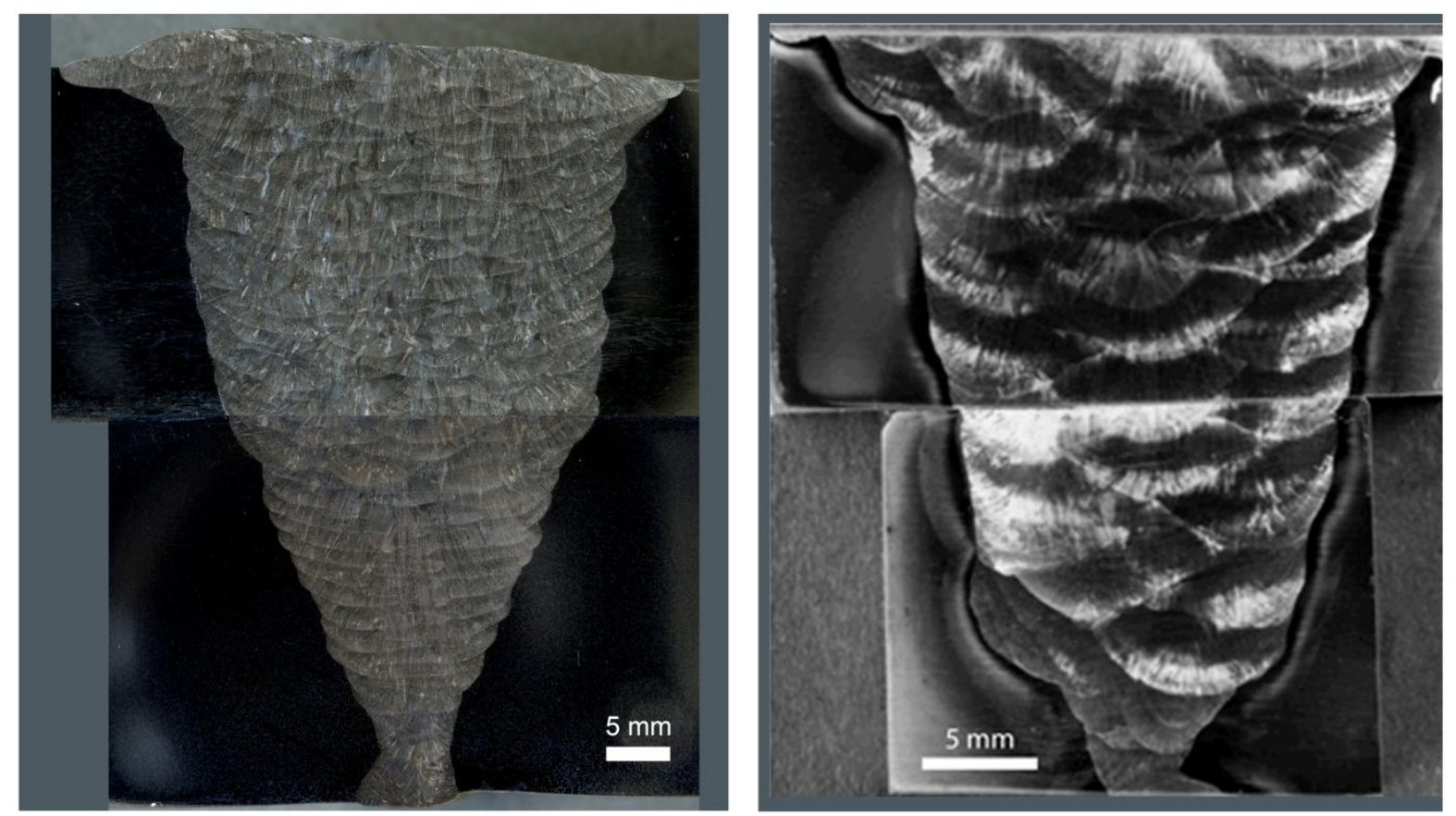

Figure 1. Macrographs of the a) austenitic RR TIG and b) ferritic EON MMA weld sections.

The sample preparation, sectioning, grinding and polishing, was only required for the EBSD measurements. The same samples were used for SRAS but that technique can be performed on much less prepared (and larger) samples. The surfaces must only be flat and shiny (mirror finish, Ra $\leq 0.1 \mu \mathrm{m}, \sim 1000$ grit polish) and must fit within the 300 $\mathrm{mm}$ by $300 \mathrm{~mm}$ range of the scanning stage.

\subsection{EBSD orientation measurements}

Crystal orientation maps were measured by EBSD (Mark, 2014) in a Camscan FEGSEM; see Figure 2. The region that can be mapped by scanning the beam in a scanning electron microscope is relatively small. For each weld section, as many as 240 individual $0.9 \times 1.2 \mathrm{~mm}^{2}$ EBSD maps were measured at a point spacing of $25 \mu \mathrm{m}$. These areas were then stitched together to make a composite map of the entire weld. This was a time-consuming process; each area took about 2 minutes to measure given a total acquisition time of $\sim 14$ hours for the EON weld and $\sim 45$ hours for the RR weld, neglecting the specimen preparation and SEM set-up times. It was also a labourintensive process as the specimen had to be manually moved to measure each new area and each time the focus had to be adjusted to maintain the best measurement conditions. It can be noted that more recently-developed EBSD acquisition systems can 
acceptably perform automatic re-focusing, which would speed up the data acquisition (Davut, 2012; Oxford Instruments, 2017).

Data analysis to determine crystal orientation is performed automatically by the EBSD software (HKL CHANNEL5, Oxford Instruments) (Schwarzer, 2009). A rejection criterion to indicate non-indexed pixels of a mean angular deviation (MAD) greater than 3.0 was used, which resulted in approximately $90 \%$ of pixels being successfully indexed.

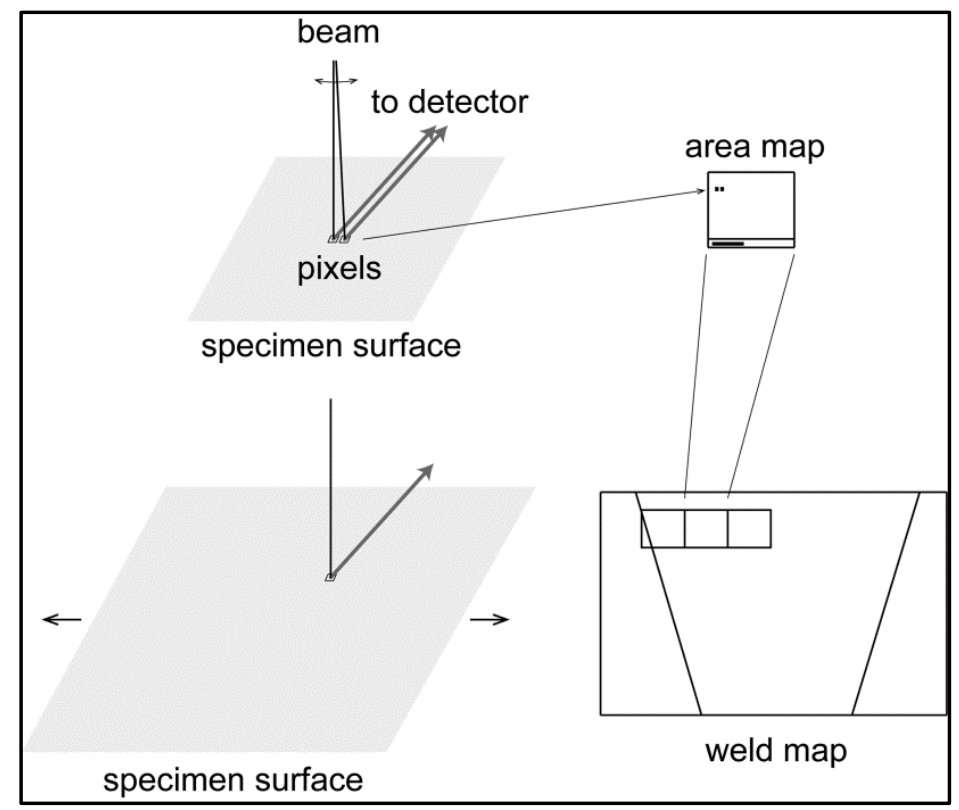

Figure 2. Schematic showing EBSD data collection process, from (Mark, 2014). The beam is automatically stepped across each area. Each step gives one data point, or one pixel in an area map. The sample is moved to acquire multiple area maps to cover the whole weld cross section.

\subsection{SRAS measurements}

The SRAS instrument, see Figure 3, is described in detail by Smith et al (Smith, 2014). In brief, the generation laser illuminates the grating, which is imaged on to the surface of the material, in $1.2 \mathrm{~ns}$ pulses at a repetition rate of $2 \mathrm{kHz}$. The optics are such that the generation patch size (the image of the grating) is $200 \mu \mathrm{m}$ in diameter. The detection spot $(\varnothing=8 \mu \mathrm{m})$ is focused on the sample surface with the same lens as is used for the generation laser and is approximately one fringe spacing distance from the edge of the generation patch. The detected signal is amplified and filtered and the trace is recorded directly on an oscilloscope to be sure a signal is recorded for each generation laser pulse. In this study the scan rate was approximately 1000 points per second including fly-back time for the raster scanning. Meanwhile the material under the generation patch is being moved at constant velocity so the location of each measurement point can be determined relative to a known start point. 
a

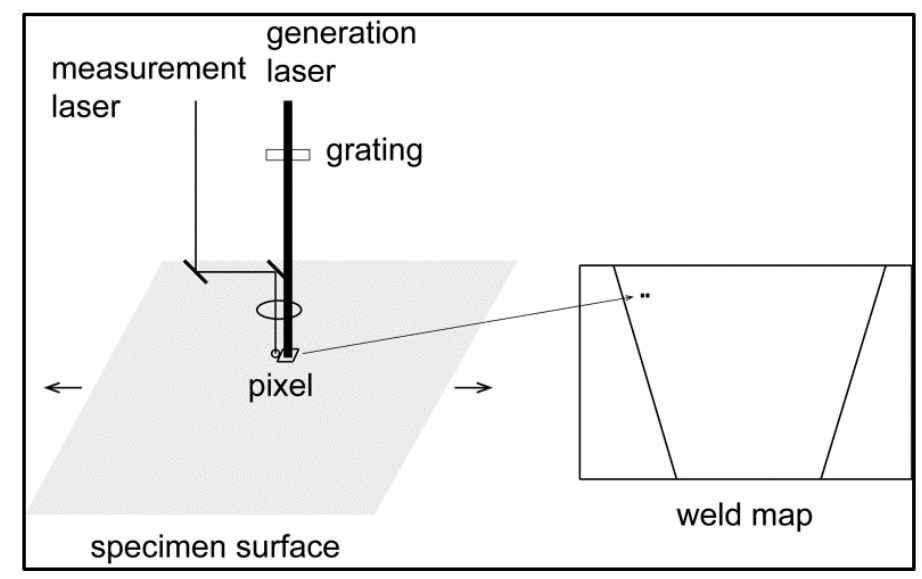

Figure 3. a) Schematic showing SRAS method and b) photo of the SRAS equipment used in this study, from (Smith, 2014).

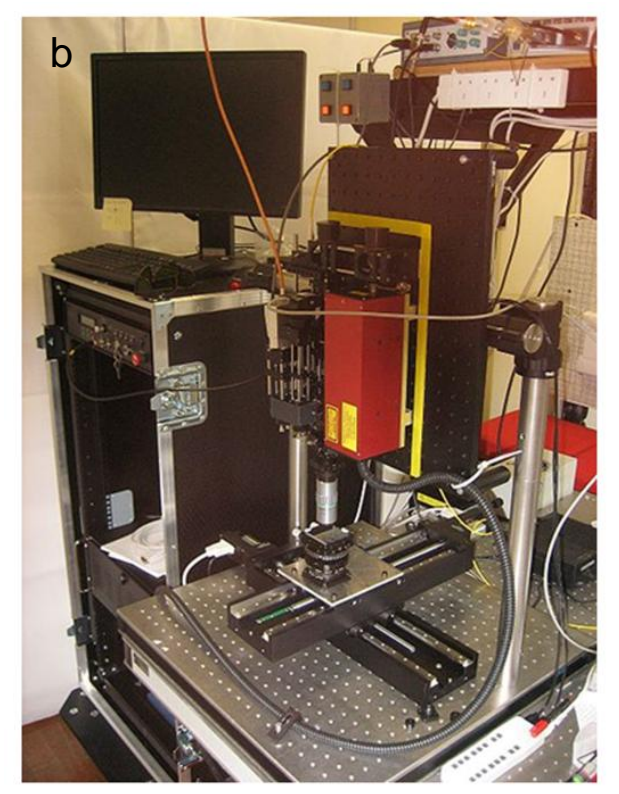

It is important to note that the measured surface acoustic wave (SAW) corresponds to the area under the image of the grating, not the point at which the SAW is detected. Since this excitation 'point' is really an area, the spatial resolution of the measurement is determined by the size of the generation patch and the power distribution in the laser. As long as the patch size is suitable for the velocity ranges measured, the velocity transition at a grain boundary is a convolution of the Gaussian distribution of the laser power and the sharp edge of the boundary. This results in a spatial resolution equal to approximately half the patch size (Smith, 2014).

The velocity resolution is determined by the number of lines in the grating pattern with more lines giving a sharper peak in the frequency profile. In practice this means that a larger patch size gives better velocity resolution, but at the expense of spatial resolution. The balance is chosen based on the expected grain size of the material (the desired spatial resolution) and considerations of the frequency bandwidth measureable by the recording electronics.

With an appropriate material model the grain orientations can be determined from the SAW data (Li, 2012; Sharples, 2012). This arises from the relationship between crystallographic orientation, crystal stiffness and SAW velocity. In theory, if one knows two of these properties the third can be calculated. In practice, put simply, we use the known crystal elastic constants given in Table 1, and rotate them from the crystal coordinate system to the bulk coordinate system. Boundary conditions are imposed such that the displacements of points at the free surface satisfy both elastic medium stress and acoustic wave equations. Then we calculate the expected SAW velocities in specific directions for a wide range of grain orientations using an iterative procedure. 
The resulting velocity surfaces are then compared to measured SAW velocity surfaces. The best match to the measured SAW velocities gives the correct orientation. A rejection criterion based on the degree of agreement between the experimental data and the calculated model resulted in rejection of the results for approximately $6 \%$ of the pixels. The degree of agreement for each pixel was mapped to an output value matrix and the pixel result was rejected if the value was below $30 \%$ of the maximum value. The method is described in more detail by $\mathrm{Li}$ et al ( $\mathrm{Li}, 2012)$. The angular resolution of the resulting orientation map depends on the number of measured directions used to build up the experimental velocity surface. Li et al describe a 2012 study of nickel; when only 6 directions were used $80 \%$ of the orientations had errors $<8^{\circ}$, whereas when 36 directions were used $80 \%$ had errors $<5^{\circ}$ ( $\left.\mathrm{Li}, 2012\right)$. By 2014 , Smith et al had achieved a standard deviation of $1.5^{\circ}$ on orientations within a single grain of aluminium (no. of measured directions not given) (Smith, 2014).

In using single crystal elastic constants the method does not take into account the constraints imposed by the neighbouring, differently oriented grains within the polycrystal. The velocities are simply calculated for an individual grain oriented in various ways relative to the sample coordinates. Symmetry arises from the crystal structure, in this case cubic; other structures give different patterns. It is also possible to start with a known polycrystal stiffness tensor, which is also rotated relative to the sample. For example, if the material is known to have a fibre texture with an unknown fibre orientation the calculated or measured stiffness tensor for a polycrystal of the relevant material with fibre texture can be used to calculate the velocity surfaces for comparison with measured velocities.

Also, it can be the case that more than one wave mode is generated for the material. In this case, the dominant wave mode is determined considering two factors: the wave must have significant displacement normal to the sample surface and it must be efficiently generated by the excitation laser (Li, 2012). The pragmatic method currently used to select the dominant mode is to calculate the displacement along the propagation direction and normalise the modulus (of the displacement) and then the mode with the largest amplitude in the plane of propagation is selected as the dominant mode for that generation direction.

Table 1. Single crystal elastic constants and densities used for SAW velocity models.

\begin{tabular}{lcccc}
\hline & $\mathrm{C}_{11}[\mathrm{GPa}]$ & $\mathrm{C}_{12}[\mathrm{GPa}]$ & $\mathrm{C}_{44}[\mathrm{GPa}]$ & Density $\left[\mathrm{g} / \mathrm{cm}^{3}\right]$ \\
\hline $\begin{array}{l}\mathrm{R} R \\
(\text { Adams, 2006) }\end{array}$ & 230 & 134 & 116 & 7.874 \\
\hline $\begin{array}{l}\text { EON - nickel } \\
\text { (Hearmon, 1956) }\end{array}$ & 247 & 153 & 122 & 8.912 \\
\hline
\end{tabular}




\section{Results}

\subsection{RR TIG Weld}

The orientation maps arising from the EBSD and SRAS measurements are shown in Figure 4 and show excellent general agreement. These show that, generally, the individual weld beads are not clearly defined but that the grain growth directions are discernible. The grain structure loses its pattern of elongated grains in the midpoint (vertically) (e.g. the region/inset marked by A) because there was a flaw introduced into the weld at this point. The continuity of the thermal gradient was broken and small grains, similar to those seen at the root of the weld, result. For the RR specimen the large vertical grain at the top right $(\mathrm{B})$ is discernible in both maps, as is the twisting of the growth in the upper middle right section $(\mathrm{C})$. The narrow vertical grains in the centre area of the bottom and the lower middle right section (D) are also visible in both sets of results. The local texture varies across the weld but it is clear that $a<100>$ direction is predominantly aligned with the solidification direction as one would expect from the weld solidification process. Unsurprisingly, given the very large number of points at which EBSD data were acquired, the EBSD map captures fine details better than the SRAS map (see insets in Figure 4). The SRAS map is noisy at the edges of the weld; areas that are shown as multiple small grains in the EBSD map appear as areas of mixed orientations in the SRAS map. Nonetheless the grain growth patterns are discernible and the general trend of larger grains in the centre of the weld and smaller grains at the boundary with the parent metal is clear and in excellent agreement with the EBSD map.

The colours in the parent material are different in the EBSD and the SRAS maps because the grain size (on the order of $60 \mu \mathrm{m}$ ) in the parent material is smaller than the SRAS spatial resolution $(\sim 100 \mu \mathrm{m})$. The SRAS measured the average velocity in this area and so the measurement cannot be interpreted in terms of crystal orientations with a single crystal model. 


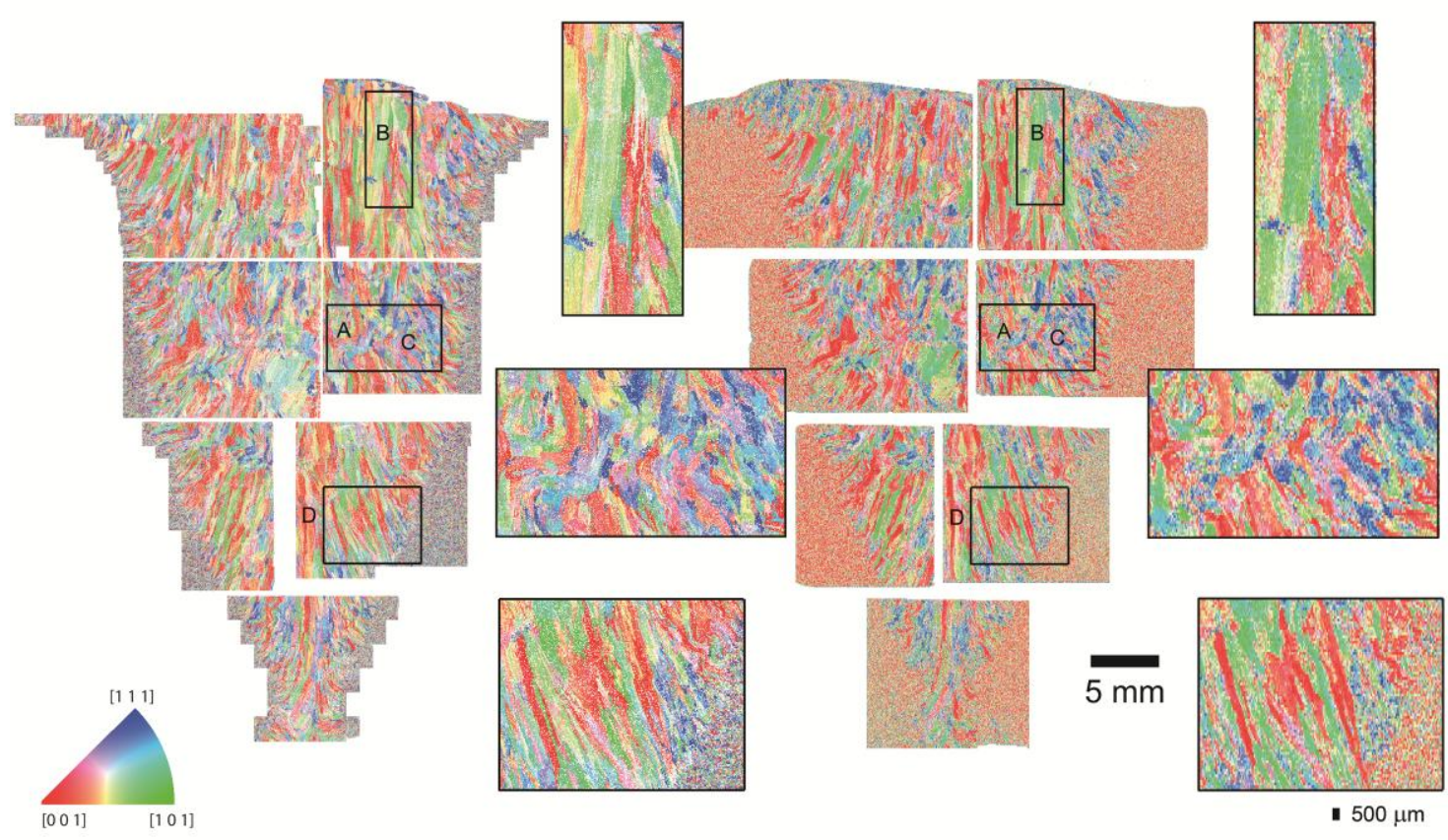

Figure 4. Grain orientation maps for the RR TIG weld specimen obtained by a) EBSD and b) SRAS along with magnified insets. The letters mark various regions of interest in the maps, see text for details.

\subsection{EON MMA Weld}

The orientation maps arising from the EBSD and SRAS measurements are shown in Figure 5 and again show excellent overall agreement. The SRAS results show lower spatial resolution compared to the EBSD results, but there are many features in common. Both maps show the large grains in the centre of both top (marked with $A$ ) and bottom ( $B$ and inset) sections. Both maps indicate a region of finer features in the right half of the lower section (below $C$ and inset). The EBSD results, however, show the structure within the region of fine features (fine, elongated grains growing approximately perpendicularly to the weld boundary), while the SRAS results appear noisy in this region as the grains are narrower than the resolution of the measurements (grains are $\sim 50 \mu \mathrm{m}$ wide) and so again the single crystal model does not obtain a good fit. 


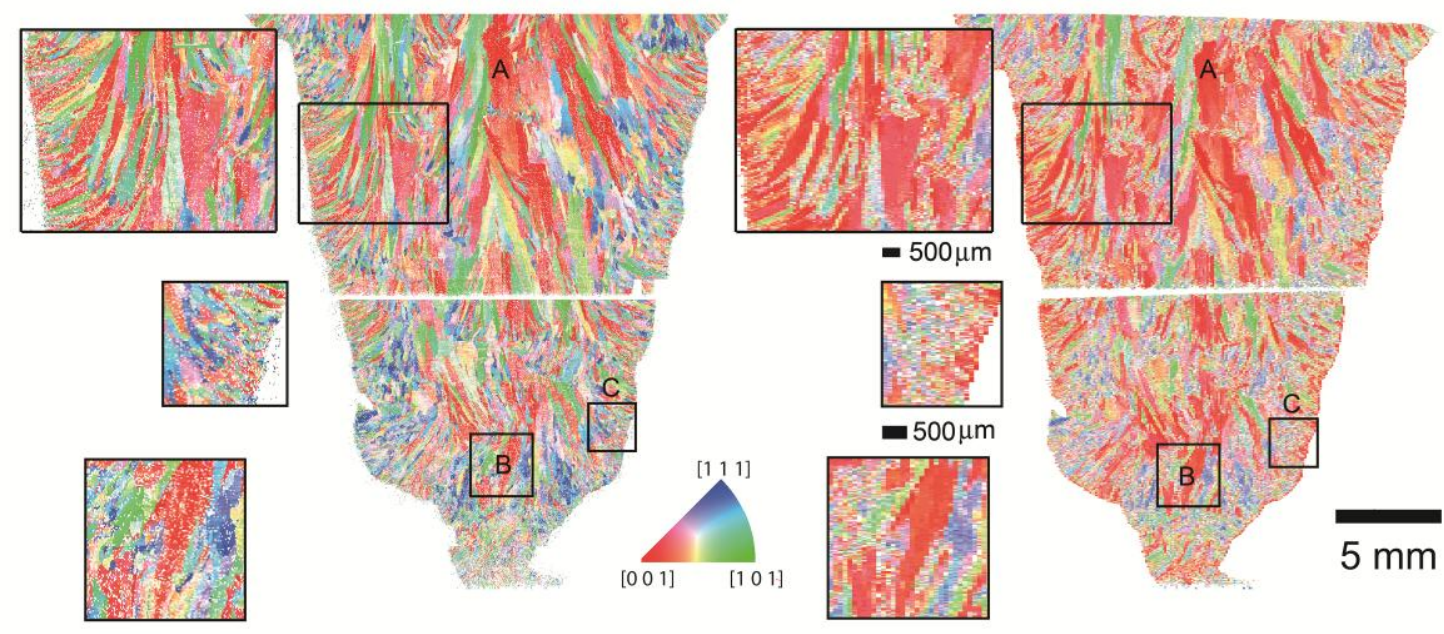

Figure 5. Grain orientation maps for the EON MMA weld specimen obtained by a) EBSD and b) SRAS along with magnified insets. The letters mark various regions of interest in the maps, see text for details.

\section{Discussion}

\subsection{Comparison of EBSD and SRAS measurements}

Evidently the EBSD and the SRAS methods give very similar results for both types of weld. As expected the SRAS maps are more pixelated and noisy than the results from EBSD in terms of defining and mapping the grain structure. The SRAS measurement method is significantly faster (4.8 hours per weld) for the large ( $30 \mathrm{~mm} \times 30 \mathrm{~mm}$ ) maps than EBSD (20 hours for a $30 \mathrm{~mm} \times 30 \mathrm{~mm}$ weld) but the latter has much better spatial resolution (here $\sim 25 \mu \mathrm{m}$ (given the measurement spacing) compared to $\sim 100 \mu \mathrm{m}$ for SRAS). See Table 2 for an overview comparison of the methods. Unfortunately our algorithm for calculating the grain orientations from the velocity measurements is run post-scanning and is not optimised for speed, but this was true of the EBSD algorithms in the early stages of development and the analysis could certainly be accelerated.

It is noteworthy that SRAS requires far less careful specimen preparation than EBSD; indeed SRAS can even be carried out on specimens with a thin coating layer $(\leq 100$ $\mathrm{nm}$ ). SRAS also has the advantage that large samples can be mapped, whereas the welds needed to be cut up for EBSD analysis because of the constraints of the SEM. Indeed, practically any size of sample can be analysed with SRAS and, with a modified measurement set-up, it would even be possible to analyse some components in-situ (e.g. fan blades or nuclear welds) in some cases. 
Table 2.

Comparison of EBSD and SRAS parameters to obtain a grain orientation map.

\begin{tabular}{|c|c|c|c|c|c|c|}
\hline Method & Weld size & $\begin{array}{l}\text { No. of meas. } \\
\text { directions }\end{array}$ & $\begin{array}{c}\text { Spatial } \\
\text { resolution }\end{array}$ & $\begin{array}{c}\text { Angular } \\
\text { resolution }\end{array}$ & $\begin{array}{l}\text { Meas. } \\
\text { time }\end{array}$ & $\begin{array}{c}\text { Analysis } \\
\text { time }\end{array}$ \\
\hline EBSD & $\begin{array}{l}\text { (RR) } 35 \mathrm{~mm} x \\
60 \mathrm{~mm}\end{array}$ & 1 & $25 \mu \mathrm{m}^{*}$ & $0.5^{\circ}$ & 45 hours & 10 hours \\
\hline EBSD & $\begin{array}{l}\text { (EON) } 25 \mathrm{~mm} x \\
27 \mathrm{~mm}\end{array}$ & 1 & $25 \mu \mathrm{m}^{*}$ & $0.5^{\circ}$ & 14 hours & 4 hours \\
\hline SRAS & $\begin{array}{l}\text { (RR) } 35 \mathrm{~mm} \mathrm{x} \\
60 \mathrm{~mm}\end{array}$ & 18 & $100 \mu \mathrm{m}$ & $1.5^{\circ}$ & 7 hours & 31 hours \\
\hline SRAS & $\begin{array}{l}(\mathrm{EON}) 25 \mathrm{~mm} x \\
27 \mathrm{~mm}\end{array}$ & 18 & $100 \mu \mathrm{m}$ & $1.5^{\circ}$ & 5 hours & 24 hours \\
\hline
\end{tabular}

*Determined by selected point spacing.

\subsection{Use of orientation data for stiffness maps for ultrasonic signal correction}

While the EBSD method relies on diffraction from the crystal lattice planes, SRAS samples the elastic stiffness directly and hence in the former case you infer the anisotropy in stiffness from the crystal orientation and in the latter it is the other way around. In practice, both methods provide a much more detailed map of the elastic anisotropy than is necessary for correcting the ultrasonic inspection process for welds. The data can be averaged to give effective elastic polycrystalline anisotropy tensors representative of sub-regions of the weld of a selected, appropriate size to create a pixelated map having the granularity required for ultrasonic signal correction. Taking the EON weld as an example, the variation in elastic stiffness tensor, represented by the direction and magnitude of the minimum in-plane stiffness, is mapped in Figure 6 for both EBSD and the SRAS measurements.

For the purposes of ultrasonic signal correction it is the variation in the direction of the 'fast (stiff)' and 'slow (compliant)' directions that matters. In Figure 6c the difference in the alignment of the compliant directions as measured by SRAS and EBSD is shown. The EBSD and SRAS maps agree within 25 degrees over the majority of the area. 


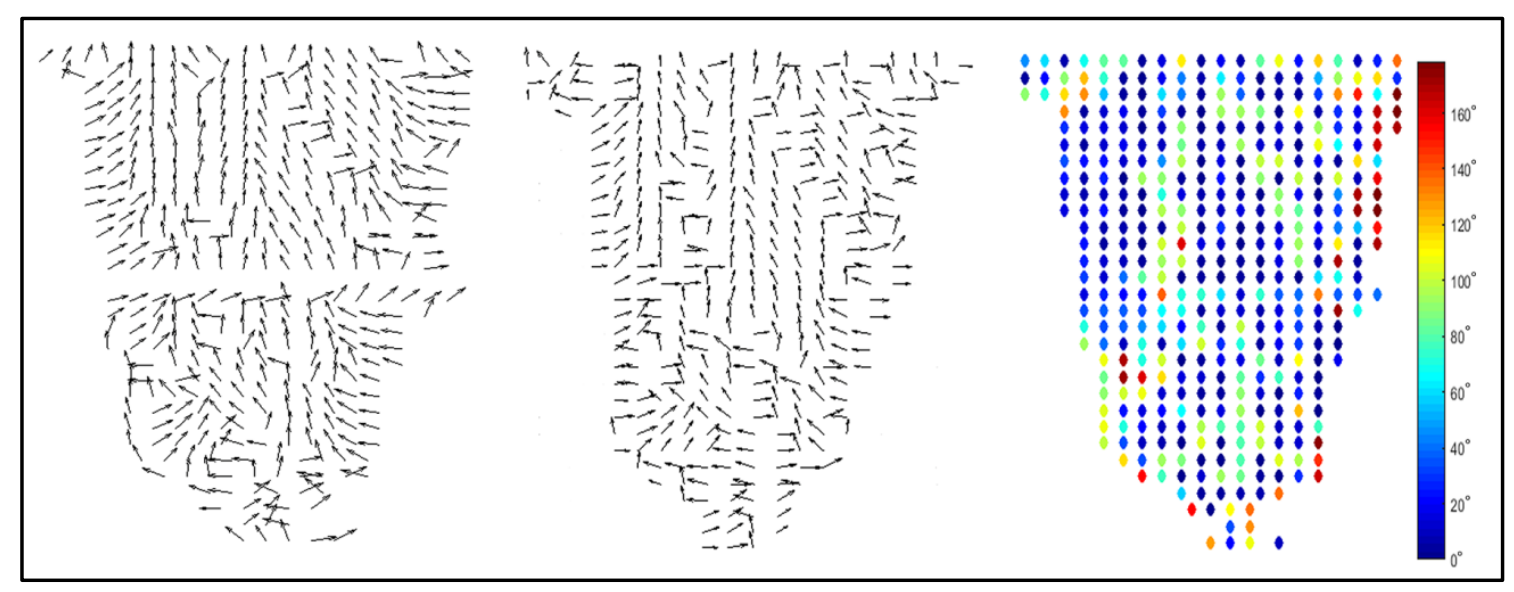

Figure 6. Maps of the direction of $E_{\min }$ for the EON weld a) as inferred from the crystal orientations from EBSD and b) from SRAS; c) shows the angle between the $E_{\min }$ directions in the maps in $a$ ) and b).

Equally, all the grain orientations in each area can be shown as local pole figures or orientation distribution functions. The variation in the local texture across the EON weld is shown in Figure 7 from the EBSD and SRAS measurements. From these measurements it is clear that the $<100>$ direction is tilted slightly out of the cross-section of the weld. In the pole figures the peak intensities are about 14.5 degrees off the plane. This is because of the effect of the movement of the hot fusion zone down the weld (Mark, 2014).

The main difference between the pole figures from the EBSD and the SRAS data is the distribution of the intensities. The EBSD pole figures show a generally transversely isotropic texture - one $<100>$ peak roughly in-plane, the preferential growth direction, and a distribution of intensity perpendicular to that. The SRAS pole figures do not have the out-of-plane angular resolution to show the perpendicular distribution. The in-plane $<100>$ peaks match those in the EBSD pole figures well but the perpendicular directions are clustered. For ultrasonic signal correction the in-plane orientation is most important so the agreement of the SRAS data on this point is convenient. 


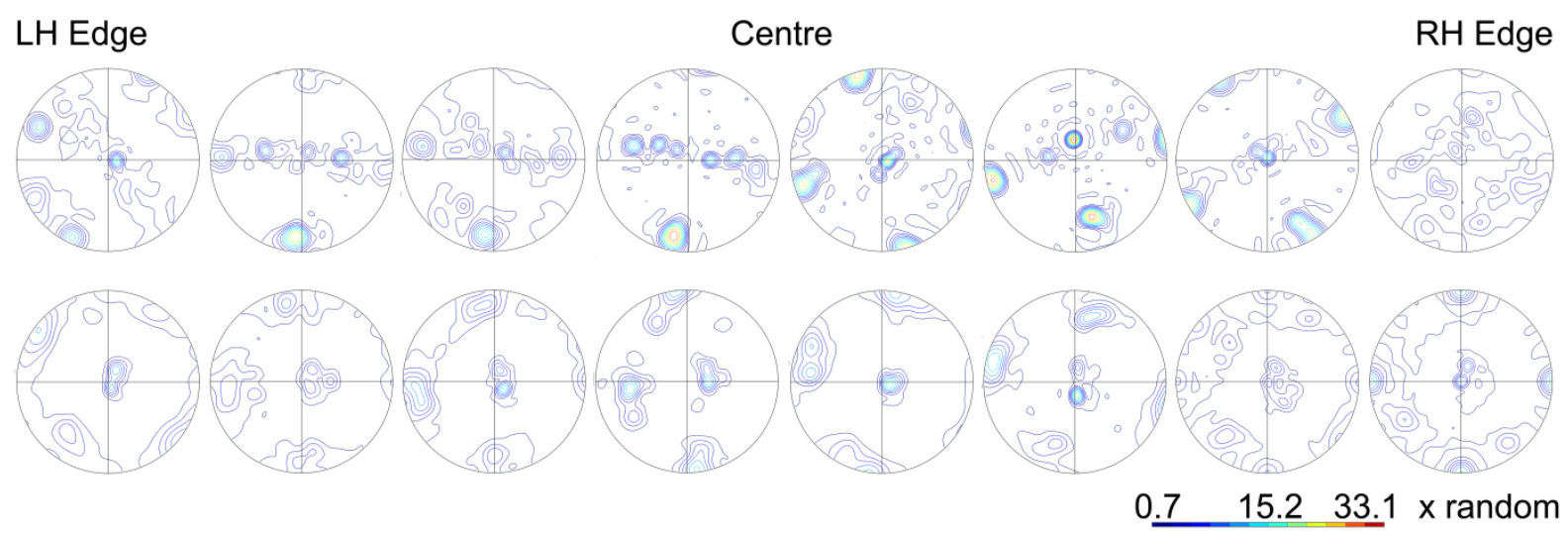

Figure 7. The variation in the local texture across the EON weld as represented by $<100>$ pole figures, measured by EBSD (top row) and SRAS (bottom row).

\section{Conclusions}

It has been verified that SRAS is a suitable method for producing grain orientation maps and that stiffness maps produced from SRAS data are in good agreement with those produced from EBSD data.

The advantages of the SRAS method are as follows:

1. In contrast to EBSD (final step $0.06 \mu \mathrm{m}$ colloidal silica) very little surface preparation is required; the surface must simply be flat and shiny (mirror finish, $\mathrm{Ra} \leq 0.1 \mu \mathrm{m})$.

2. Large samples can be accommodated; one is only limited by the space available under the laser system. Measurements can even be done in-situ with an appropriately mounted laser set-up.

3. The measurements are quick ( 4.8 hours for $30 \mathrm{~mm}$ by $30 \mathrm{~mm} ; 5$ times faster than EBSD) and require minimal (no) intervention once begun. This is in comparison with EBSD measurements where the maps in Figures 4 and 5 had to be assembled by stitching together hundreds of individual EBSD maps. Each required re-focusing. The re-focusing, added to the longer individual measurement time for EBSD, mean that $\sim 20$ hours are required to obtain equivalently-sized maps. Again it is noted that more recently-developed automated EBSD systems can acceptably perform this re-focusing, somewhat lessening the time advantage for SRAS.

The current implementation of the SRAS method compares unfavourably to EBSD in the following respects:

1. The spatial resolution of SRAS is around $(\sim 100 \mu \mathrm{m})$. This is not as good as that for EBSD used here $(\sim 25 \mu \mathrm{m})$ and far inferior to that achieved at the limit (a few 
$\mathrm{nm})$. In practice the ability to accurately infer the crystal orientations is related to the degree of elastic anisotropy - the greater the anisotropy the better the discrimination.

2. The angular resolution is $\sim 1.5^{\circ}$ (Smith, 2014), which compares with around $0.5^{\circ}$ for EBSD.

3. Data analysis is computationally intensive and iterative. It requires calculation of velocity surfaces for many orientations and comparison of each to the experimental data until the best match is found. This is considered a brute force approach; it is not computationally challenging but it is resource intensive and slow. This can easily be accelerated through optimisation.

Overall, the stiffness maps produced using SRAS are in good agreement with those produced from EBSD data (Figure 7). Previous work has shown that stiffness maps from EBSD data are of more than sufficient resolution to allow refinement of model stiffness maps used to correct ultrasonic flaw detection signals (Mark, 2014). Since the routines used to produce these model stiffness maps involve iterative correction, the requirements for the initial input maps are not stringent (Fan, 2014). Angular agreement within 25 degrees is acceptable so SRAS maps could be used as input data. In fact, the lower spatial and angular resolutions of the SRAS measurements are not impediments to their use for ultrasonic signal correction. The SRAS resolution is sufficient.

Improvements in speed, both of measurement and analysis, that are the subject of current work will make the SRAS method even faster, and achievable improvements in detection electronics will allow higher-resolution measurements (Smith, 2014). Given that ultrasonic inspection of welds is generally used for industry, where the process and the correction needs to be quick, easy to implement, and low cost, SRAS stiffness mapping is the logical choice for signal correction.

And it does not end there; the options for other applications of SRAS are intriguing and plentiful. For example, it could be used to deduce the presence of rogue grains in single crystal turbine blades, or to map the texture of fan blades or of sheet metals during processing. Once the equipment has been made practical for use on minimally processed engineering components, and in-situ within a manufacturing environment, the SRAS method will certainly become a standard inspection method in a plethora of industries.

\section{Acknowledgements}

We would like to acknowledge funding from EPSRC via grants EP/G042772/1, EP/G045720/1 and EP/G061661/1. We would also like to acknowledge contributions from the UK Research Centre in Non-Destructive Evaluation (RCNDE), Rolls-Royce Nuclear and E.ON. We would like to thank David Fan, Mike Lowe, Colin Brett, David Duxbury, and Alan Catchpole for their valuable advice. Underlying research materials for this paper can be accessed by contacting the corresponding author (AFM). 


\section{References}

(Adams, 2006) J.J. Adams, D.S. Agosta, R.G. Leisure, "Elastic constants of monocrystal iron from 3 to 500 K”, J. Appl. Phys. 100 (2006) 113530.

(Davut, 2012) K. Davut, S. Zaefferer, "Improving the Reliability of EBSD-based Texture Analysis by a New Large Area Mapping Technique", Mater. Sci. Forum 702-703 (2012) 566-569.

(Dingley, 1992) D.J. Dingley, V. Randle, "Review Microtexture determination by electron back-scatter diffraction”, J. Mater. Sci. 27 (1992) 4545-4566.

(Fan, 2013) Z. Fan, M.J.S. Lowe, "Characterization of inhomogeneous and anisotropic steel welds by ultrasonic array measurements", AIP Conf. Proc. 1511 (2013) 849-856.

(Fan, 2014) Z. Fan, A.F. Mark, M.J.S. Lowe, P.J. Withers, "Nonintrusive Estimation of Anisotropic Stiffness Maps of Heterogeneous Steel Welds for the Improvement of Ultrasonic Array Inspection", IEEE T. Ultrason. Ferr.62 (2015) 1530-1543.

(Hearmon, 1956) R. Hearmon, "The elastic constants of anisotropic materials - ii", Adv. Phys. 5 (1956) 323-382.

(Li, 2012) W. Li, S.D. Sharples, R.J. Smith, M. Clark, M. Somekh, "Determination of crystallographic orientation of large grain metals with surface acoustic waves", J. Acoust. Soc. Am. 132 (2012) 738-745.

(Li, 2016) W. Li, J. Coulson, P. Marrow, R.J. Smith, S.J. Laine, M. Clark, S.D. Sharples, "Crystallographic Orientation Determination of Hexagonal Structure Crystals by Laser Ultrasonic Technique”, J. Phys.: Conf. Series 684 (2016) 012001.

(Mark, 2014) A.F. Mark, Z. Fan, M.J.S. Lowe, P.J. Withers, "Investigation of the elastic/crystallographic anisotropy of welds for improved ultrasonic inspections", Mater.Charact.98 (2014) 47-53.

(Oxford Instruments, 2017) EBSD Large Area Mapping, https://www.oxfordinstruments.com/products/microanalysis/ebsd/aztechkl-ebsd-software, accessed 2-22017.

(Schwarzer, 2009) R.A. Schwarzer, D.P. Field, B.L. Adams, M. Kumar, A.J. Schwartz, "Present State of Electron Backscatter Diffraction and Prospective Developments" in Electron Backscatter Diffraction in Materials Science, A.J. Schwartz, M. Kumar, B.L. Adams, D.P. Field, eds., Springer, New York, 2009.

(Sharples, 2012) S.D. Sharples, W. Li, R. Smith, J. Coulson, M. Clark, M. Somekh, "SRAS Characterisation Capabilities, and Where We Go From Here", presented at RCNDE SRAS Workshop, University of Nottingham, 8 March 2012. 
(Smith, 2014) R.J. Smith, W. Li, J. Coulson, M. Clark, M.G. Somekh, S.D. Sharples, "Spatially resolved acoustic spectroscopy for rapid imaging of material microstructure and grain orientation”, Meas. Sci. Technol. 25 (2014) 055902. 